\title{
A retrospective audit of the provision of PEPSE In a community sexual health clinic
}

Dr Sarah Clark, FY2 Community Sexual and Reproductive Health, Mr Dale Coley, IT Analyst,

Dr Barbara Vonau, Locum Consultant in Genitourinary Medicine Dr Eleanor Draeger, Consultant in Genitourinary Medicine. Department of Sexual and Reproductive Health, Lewisham and Greenwich NHS Trust

\section{Introduction}

The 2011 British Association for Sexual Health and HIV (BHIVA) UK guidelines for the use of post-exposure prophylaxis for HIV following sexual exposure (PEPSE) outlines clear recommendations for the use of PEPSE 1 The guidelines outline six auditable outcomes:

British Association for Sexual Health and HIV, London, UK: UK guideline for the use of post-exposure prophylaxis for HIV following sexual exposure (2011)P Benn, M Fisher, R Kulasegaram

PEPSE Guidelines Writing Group Clinical Effectiveness Group

http://www.bhiva.org/documents/guidelines/pepse/pepse2oll.pdf

The provision of PEPSE at Lewisham and Greenwich NHS had been such that the assessment and initiation of PEPSE was located at the SRH hub in the community in New Cross (Waldron Centre), while follow up was at the HIV care centre (Alexis Clinic) at University Hospital Lewisham, which is approximately 45 minutes away if travelling on public transport.

Six Auditable Outcomes outlined in BHIVA PEPSE guidelines 2011:

Proportion of PEPSE patients having a baseline HIV test: aim 100\% within 72 hours of presenting for PEPSE;

Proportion of PEPSE prescriptions that fit within recommended indications: aim $90 \%$

Proportion of PEPSE prescriptions administered within 72 hours of risk exposure: aim $90 \%$;

Proportion of individuals completing 4-week course of PEPSE: aim 75\%;

Proportion of individuals seeking PEPSE undergoing testing for STIs: aim 90\%;

Proportion of individuals completing 12-week post-PEP HIV antibody/ antigen test: aim $60 \%$

\section{Aims}

To assess the extent to which the auditable outcomes, outlined in the 2011 BHIVA guidelines have been achieved when assessing the need for PEPSE, prescribing PEPSE at the Waldron Centre, London and follow up for completion and STI/HIV testing.

\section{Methods}

Electronic records from The Waldron Centre, London, were retrospectively analysed for consultations which had been coded as 'PEPSE' between January 2013 and July 2015. Follow up data was collected from the electronic records from both The Waldron Centre, London and The Alexis Clinic, London, and the paper records from the Alexis clinic, were follow up was performed. Data was collected for each of the auditable outcomes outlined in the 2011 BHIVA PEPSE guidelines.

Using combined diagnoses as reference standard, microscopy was 100\% sensitive for trichomonas vaginalis (TV), $97 \%$ sensitive for bacterial vaginosis (BV) and $85 \%$ sensitive for vulvo-vaginal candida albicans (VVC). This (BV) and $85 \%$ sensitive for vulvo-vaginal candida albicans (VVC). This have missed $4(50 \%)$ of the TV cases and $50(82 \%)$ of the BV cases. Using microscopy alone would have missed 2 (100\%) cases of Group B Strep, $2(3 \%)$ cases of BV and $4(15 \%)$ cases of VVC. GNICD were seen on microscopy on 2 occasions. Neither of these 2 women had gonorrhoea neisseria proved on HVS or endocervical NAAT. In 2 other cases chlamydia trachomatis was found on endocervical NAAT.

\section{Sample:}

After excluding patients who presented with percutaneous exposure and those who declined PEPSE we were left with a sample of 72 patients. 68 were male and 60 were men who have sex with men.

\section{Results:}

\begin{tabular}{|l|l|l|}
\hline Outcome & Percentage (\%) & BHIVA aim (\%) \\
\hline HIV testing within 72hrs baseline & $100 \%$ & $100 \%$ \\
\hline Prescription fitting indication & $100 \%$ & $90 \%$ \\
\hline PEPSE within 72 hours & $100 \%$ & $90 \%$ \\
\hline Completion of 4 weeks of PEPSE & 29.2 & $75 \%$ \\
\hline Complete STI screen & $80.6 \%$ & $90 \%$ \\
\hline $\begin{array}{l}\text { HIV testing 12 weeks post PEP } \\
\text { completion }\end{array}$ & $25 \%$ & $60 \%$ \\
\hline
\end{tabular}

\section{Discussion:}

The recommended outcomes were easily achieved for completion of a baseline HIV test, prescribing PEPSE in accordance with the guidelines and commencing PEPSE within 72 hours. These are all factors which generally take place at the initial appointment.

However, we fell short of the BHIVA aims for the factors which required follow up at a later date; completion of 4 weeks of PEPSE, completion of a ful STI screen and an HIV test 12 weeks post completion.

This demonstrates the successful initiation of PEPSE in a inner city community sexual health clinic. However, the follow up targets were not met, which may be explained by the fact that follow up is provided at a separate location. Potential causes include travel issues, and a lack of fail safe management of DNAs in a busy HIV clinic.

management of DNAs in a busy HIV clinic.
Following these findings, PEPSE follow up has been relocated in March Following these findings, PEPSE follow up has been relocated in March
2017 to the Waldron Clinic in an attempt to reduce the loss to follow up. A PEPSE management pathway has been introduced as well as a template on the Electronic patient record used to prompt adequate data collection for each PEPSE related visit. In the first three months after this change we have supplied 10 patients with PEPSE.

A repeat audit is planned in approximately 12 months using the revised 2015 guidelines2 auditable outcomes (Due to an update in BHIVA guidelines).

\section{References:}

1. British Association for Sexual Health and HIV, London, UK: UK guideline for the use of post-exposure prophylaxis for HIV following sexual exposure (2011)P Benn, M Fisher, R Kulasegaram. PEPSE Guidelines Writing Group Clinical Effezctiveness Group. www.bhiva.org/documents/guidelines/pepse/pepse2ol1.pdf

2. British Association for Sexual Health and HIV, London, UK: UK guideline for the use of post-exposure prophylaxis for HIV following sexual exposure (2015) M Fisher et al: www.bashh.org/documents/PEPSE\%202015\%20guideline\%201inal_NICE.pdf

Contact: barbaravonau@nhs.net, eldraeger@gmail.com 